\title{
Analytical Wave Solutions for Foam and KdV-Burgers Equations Using Extended Homogeneous Balance Method
}

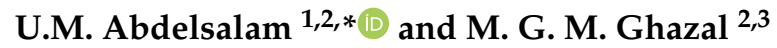 \\ 1 Department of Mathematics, Faculty of Science, Fayoum University, Fayoum 63514, Egypt \\ 2 Department of Mathematics, Rustaq College of Education, Ministry of Higher Education, Rustaq 329, Oman \\ 3 Department of Mathematics, Minia University, Minia 61519, Egypt \\ * Correspondence: usama.ahmad@rub.de
}

Received: 8 July 2019; Accepted: 1 August 2019; Published: 9 August 2019

\begin{abstract}
In this paper, extended homogeneous balance method is presented with the aid of computer algebraic system Mathematica for deriving new exact traveling wave solutions for the foam drainage equation and the Kowerteg-de Vries-Burgers equation which have many applications in industrial applications and plasma physics. The method is effective to construct a series of analytical solutions including many types like periodical, rational, singular, shock, and soliton wave solutions for a wide class of nonlinear evolution equations in mathematical physics and engineering sciences.
\end{abstract}

Keywords: foam drainage equation; Kowerteg-de Vries-Burgers equation; extended homogeneous balance method; exact solutions; nonlinear evolution equation

\section{Introduction}

The nonlinear evolution equations and their solutions are very important for understanding many physical phenomena, for example studying the waves observed in plasma, fluids, optical fibers, laser, astrophysics, water waves and other areas of engineering and science [1-5]. There are several methods have been constructed and developed for finding the traveling wave solutions to the nonlinear partial differential equations. The more common used methods are inverse scattering [6,7], bilinear transformation $[8,9]$, the tanh-function $[10,11]$, extended tanh [12,13], expansion-function [14], $\left(G^{\prime} / G\right)$ method [15-17], general expansion [18,19] and the symmetry method [20]. The homogeneous balance (HB) method $[21,22]$ is a powerful method for deriving the exact traveling wave solutions, the HB method $[23,24]$ was extended to give different types of exact solutions. HB method was also used by Fan [25] to add two new applications for this method.

The foam drainage Equation [26] is very important to describe the drainage of liquid in a foam. In 19th century, foam was first clearly described by the Joseph plateau. Nowadays, foams have many industrial applications and play an important role in many technological processes and applications, and there are many practical and scientific studies for their properties.

The KdV-Burgers equation [27-29] is a very common and important equation in studying solitary waves and acoustic waves in plasma physics [30]. Shock waves due to thde KdV-Burgerss equation were observed in dusty plasma by Nakamura et al. [31] and Masoad et al. [32].

In this paper, an extend homogeneous balance method [3] presented to construct many types of analytical solutions for the foam drainage equation [33] and the Kowerteg-de Vries-Burgerss equation. The paper is organized as follows: in Section 2, the extend homogeneous balance (EHB) method is described. In Section 3, we apply the EHB method to solve the foam Drainage equation and the Kowerteg-de Vries-Burgers equation. The discussion and results are presented in Section 4. Finally, the results are summarized in Section 5 . 


\section{Proposed Analytical Method}

Consider the PDE (in two variables)

$$
\psi\left(\varphi, \varphi_{t}, \varphi_{x}, \varphi_{x x}, \ldots\right)=0,
$$

the traveling wave solution proposed to be in the form

$$
\varphi(x, t)=\varphi(\eta), \quad \eta=x-\lambda t
$$

where $\lambda$ is a constant. Reducing Equation (1) to an ordinary differential equation (ODE) we use the transformation (2). Now we consider the solution in the form

$$
\varphi(\eta)=\sum_{i=0}^{n} \alpha_{i} Q^{i}+\sum_{i=1}^{n} \beta_{i}[1+Q]^{-i}
$$

and

$$
Q^{\prime}=k+M Q+P Q^{2}
$$

where $\alpha_{i}$ and $\beta_{i}$ are constants, while $k, M$ and $P$ are parameters to be determined later, $Q=Q(\eta)$, and $Q^{\prime}=d Q / d \eta$. The different effects (such as, the dispersion and nonlinearity) of the partial differential equation acts to make solitary waves by changing the wave form and have to balance each other. So by balancing the highest-order linear term with the nonlinear terms we can determine the positive integer parameter $n$. Substitute (3) and (4) in the obtained ODE, this will produce a system of ODEs with respect to the last parameters, then equate all the coefficients of $Q^{j}$ (where $j=0,1, \ldots$ ) to zero, and determine $\alpha_{0}, \alpha_{i}, \beta_{i}, k, M, P$ and $\lambda$ using Mathematica.

It is to be noted that the Riccati Equation (4) can be solved using the homogeneous balance method as follows:

Case I: when $\mathrm{P}=1, \mathrm{M}=0$, the Riccati Equation (4) has the following solutions

$$
\begin{gathered}
Q= \begin{cases}-\sqrt{-k} \tanh [\sqrt{-k} \eta], & \text { with } k<0, \\
-\sqrt{-k} \operatorname{coth}[\sqrt{-k} \eta], & \text { with } k<0,\end{cases} \\
Q=-\frac{1}{\eta}, \quad \text { with } \quad k=0,
\end{gathered}
$$

and

$$
Q= \begin{cases}\sqrt{k} \tan [\sqrt{k} \eta], & \text { with } k>0, \\ -\sqrt{k} \cot [\sqrt{k} \eta], & \text { with } k>0 .\end{cases}
$$

Case II: let $Q=\sum_{i=0}^{m} \alpha_{i} \tanh ^{i}\left(p_{1} \eta\right)$. Balancing $Q^{\prime}$ with $Q^{2}$ leads to

$$
Q=\alpha_{0}+\alpha_{1} \tanh \left(p_{1} \eta\right)
$$

then, substituting Equation (8) into (4), the obtained solution for Equation (4) will be in the form

$$
Q=-\frac{p_{1}}{2 P} \tanh \left(\frac{p_{1}}{2} \eta\right)-\frac{M}{2 P}, \text { with } P k=\frac{M^{2}-p_{1}^{2}}{4}
$$

Similarly, let $Q=\sum_{i=0}^{m} \alpha_{i} \operatorname{coth}^{i}\left(p_{1} \eta\right)$, then the obtained solution for Equation (4) will be in the form

$$
Q=-\frac{p_{1}}{2 P} \operatorname{coth}\left(\frac{p_{1}}{2} \eta\right)-\frac{M}{2 P}
$$

with $P k=\frac{M^{2}-p_{1}^{2}}{4}$. 
Case III: we suppose that the Riccati Equation (4) have the following solutions of the form

$$
Q=\alpha_{0}+\sum_{i=0}^{m}\left(\alpha_{i} f^{i}+\beta_{i} f^{i-1} g\right)
$$

with

$$
f=\frac{1}{\cosh \eta+r}, g=\frac{\sinh \eta}{\cosh \eta+r},
$$

substituting Equations (10) and (11) into (4), will give the solution

$$
Q=-\frac{1}{2 P}\left(M+\frac{\sinh (\eta)+\sqrt{r^{2}-1}}{\cosh (\eta)+r}\right), \text { with } P k=\frac{M^{2}-1}{4},
$$

where $r$ is an arbitrary constant. If $r=1$ then solution (12) will be in the form,

$$
Q=-\frac{1}{2 P}\left[M+\tanh \left(\frac{\eta}{2}\right)\right]
$$

Case IV: we suppose that the solutions of Riccati Equation (4) in the form

$$
Q=\alpha_{0}+\sum_{i=0}^{m} \sinh ^{i-1}\left(\alpha_{i} \sinh \xi+\beta_{i} \cosh \xi\right)
$$

where $d \xi / d \eta=\sinh \xi$ or $d \xi / d \eta=\cosh \xi$. Balancing $Q^{\prime}$ with $Q^{2}$ leads to $m=1$

$$
Q=\alpha_{0}+\alpha_{1} \sinh \xi+\beta_{1} \cosh \xi
$$

when $d \xi / d \eta=\sinh \xi$ we substitute (15) and $d \xi / d \eta=\sinh \xi$ into (4) and set the coefficient of $\sinh ^{i} \xi \cosh ^{j} \xi, i=0,1,2, j=0,1$ to zero and solve the obtained set of algebraic equations we get

$$
\alpha_{0}=\frac{-M}{2 P}, \alpha_{1}=0, \beta_{1}=\frac{1}{P}
$$

where $k=\frac{M^{2}-4}{4 P}$ and

$$
\alpha_{0}=\frac{-M}{2 P}, \alpha_{1}= \pm \sqrt{\frac{1}{2 P}}, \beta_{1}=\frac{1}{P}
$$

where $k=\frac{M^{2}-1}{4 P}$, for $d \xi / d \eta=\sinh \xi$ we have

$$
\sinh \xi=-\operatorname{csesh} \eta, \cosh \xi=-\operatorname{coth} \eta
$$

From (16)-(18) we obtain

$$
Q=-\frac{M+2 \operatorname{coth} \eta}{2 P}
$$

where $k=\frac{M^{2}-4}{4 P}$, and

$$
Q=-\frac{M \pm \operatorname{csesh} \eta+\operatorname{coth} \eta}{2 P}
$$

where $k=\frac{M^{2}-1}{4 P}$.

\section{Applied Examples}

Now we will apply the above approach for two nonlinear evolution equations namely, the foam drainage equation and KdV-Burgers equation, which are encountered in many physical phenomena and industrial applications. It is known that KdV-Burgers equation is important in studying different 
physical systems in fluids and plasmas. Studying the properties of the foam is very important in many applications and technology.

\subsection{Example 1. Foam Drainage Equation}

We apply the extended homogeneous balance method to construct the traveling wave solutions for foam drainage equation,

$$
\varphi_{t}+\left(\varphi^{2}-\frac{\sqrt{\varphi}}{2} \varphi_{x}\right)_{x}=0
$$

Using the transformation $\varphi(x, t)=V(\eta), \eta=x-\lambda t$ to Equation (21) we get the following ODE

$$
-\lambda V^{\prime}+2 V V^{\prime}-\frac{V^{\prime 2}}{4 \sqrt{V}}-\frac{1}{2} \sqrt{V} V^{\prime \prime}=0 .
$$

On integrating (22) with respect to $\eta$ once, we get

$$
V^{2}-\lambda V-\frac{1}{2} V^{\prime} \sqrt{V}=0
$$

use transformation:

$$
V(\eta)=U^{2}(\eta) \text { we get, }
$$

$$
-\lambda+U^{2}-U^{\prime}=0
$$

Balancing $U^{2}$ with $U^{\prime}$ gives $n=1$. So the solution should be in the form

$$
U=\alpha_{0}+\beta_{0}+\alpha_{1} Q+\beta_{1}(1+Q)^{-1} .
$$

Now from Equations (4) and (24) in Equation (23), this produces a polynomial equation in $Q$. Thus, if we equate the coefficients of $Q^{s}$ (such as, $s=0,1,2, \ldots$ ) to zero, using Mathematica, we can solve the obtained system of overdetermined algebraic equations.

The first set:

$$
\alpha_{0}=\frac{M}{2}, \alpha_{1}=\frac{M}{2}, \beta_{1}=\frac{\lambda}{2 M}, k=\frac{1}{2}\left(M-2 \beta_{1}\right), P=\frac{M}{2}, M \beta_{1} \neq 0 .
$$

The second set:

$$
\alpha_{1}=0, \lambda=\frac{1}{2}\left(2 \alpha_{0}^{2}+2 \beta_{1} \alpha_{0}+M \beta_{1}\right), \beta_{1}=\frac{1}{2}\left(-2 k+M-2 \alpha_{0}\right), P=\frac{1}{2}\left(M+2 \alpha_{0}\right), M \beta_{1}+2 \alpha_{0} \beta_{1} \neq 0 .
$$

The third set:

$$
\alpha_{0}=\frac{M}{2}, \alpha_{1}=P, \beta_{1}=0, k=\frac{M^{2}-4 \lambda}{4 P} .
$$

For the first set (25) we note that the solutions can satisfy cases II-IV which need to apply the compatibility condition,

$$
P k=\frac{M^{2}-p_{1}^{2}}{4} .
$$

The last equation can be solved for $p_{1}$ by substituting for $M, k$ and $P$ from (25) to give

$$
p_{1}=\sqrt{\lambda}
$$

Therefore, the obtained solutions to foam drainage Equation (21) can be written as

$$
\varphi_{1}(x, t)=\frac{1}{2}\left(M-\sqrt{\lambda}(M+2 \tanh ((x-\lambda t) \sqrt{\lambda}))+\frac{\lambda}{-\sqrt{\lambda} M+M-2 \sqrt{\lambda} \tanh ((x-\lambda t) \sqrt{\lambda})}\right),
$$


and

$$
\varphi_{2}(x, t)=\frac{1}{2}\left(M-\sqrt{\lambda}(M+2 \operatorname{coth}((x-\lambda t) \sqrt{\lambda}))+\frac{\lambda}{-\sqrt{\lambda} M+M-2 \sqrt{\lambda} \operatorname{coth}((x-\lambda t) \sqrt{\lambda})}\right),
$$

and for case III,

$$
\varphi_{3}(x, t)=-\frac{2(\lambda+1) r^{2}+4 \lambda \cosh (x-\lambda t) r+\lambda+(\lambda+1) \cosh (2(x-\lambda t))+4 \sqrt{r^{2}-1} \sinh (x-\lambda t)-3}{4(r+\cosh (x-\lambda t))\left(\sinh (x-\lambda t)+\sqrt{r^{2}-1}\right)},
$$

with condition $p_{1}=1$, while solutions for case IV are

$$
\varphi_{4}(x, t)=\frac{1}{2}\left(2 M+\operatorname{coth}(x-\lambda t)+\operatorname{csch}(x-\lambda t)+\frac{\lambda}{2 M+\operatorname{coth}(x-\lambda t)+\operatorname{csch}(x-\lambda t)}\right),
$$

with $p_{1}=1$, and

$$
\varphi_{5}(x, t)=-\operatorname{coth}(x-\lambda t)-\frac{1}{4} \lambda \tanh (x-\lambda t)
$$

with $p_{1}=2$.

For the second set (26), we can apply for case I with $P=1$ and $M=0$, thus for $k>0$ the solutions will be

$$
\begin{aligned}
& \varphi_{6}(x, t)=1-\frac{k+1}{\sqrt{k} \tan (\sqrt{k}(x-\lambda t))+1}, \\
& \varphi_{7}(x, t)=1-\frac{k+1}{\sqrt{k} \cot (\sqrt{k}(x-\lambda t))+1} .
\end{aligned}
$$

For $k<0$,

$$
\begin{gathered}
\varphi_{8}(x, t)=1+\frac{k+1}{\sqrt{-k} \tanh (\sqrt{-k}(x-\lambda t))-1}, \\
\varphi_{9}(x, t)=1+\frac{k+1}{\sqrt{k} \operatorname{coth}(\sqrt{k}(x-\lambda t))-1},
\end{gathered}
$$

and for $k=0$, we get

$$
\varphi_{9}(\eta)=\frac{1}{1+\eta}
$$

For cases II-IV, we apply the compatibility condition with the values of $M, k$ and $P$ in (26), we get $p_{1}=2 \sqrt{\lambda}$.

Now, for case II, we obtain

$$
\varphi_{10}(x, t)=\frac{P\left(-2 k+M-2 \alpha_{0}\right)}{2 P-p_{1}\left(M+2 \tanh \left((x-\lambda t) p_{1}\right)\right)}+\alpha_{0}
$$

and

$$
\varphi_{11}(x, t)=\alpha_{0}+\frac{P\left(-2 k+M-2 \alpha_{0}\right)}{2 P-\left(M+2 \operatorname{coth}\left((x-\lambda t) p_{1}\right)\right) p_{1}}
$$

and for case III,

$$
\varphi_{12}(x, t)=\alpha_{0}+\frac{P(r+\cosh (x-\lambda t))\left(2 k-M+2 \alpha_{0}\right)}{M r-2 P r+(M-2 P) \cosh (x-\lambda t)+\sinh (x-\lambda t)+\sqrt{r^{2}-1}}
$$

with $p_{1}=1$, while for case IV,

$$
\varphi_{13}(x, t)=\frac{(M-2 k) P+(M+\operatorname{coth}(x-\lambda t)+\operatorname{csch}(x-\lambda t)) \alpha_{0}}{M+2 P+\operatorname{coth}(x-\lambda t)+\operatorname{csch}(x-\lambda t)},
$$


with $p_{1}=1$,

$$
\varphi_{14}(x, t)=\frac{(2 k-M) P+(M+2 \operatorname{coth}(x-\lambda t)) \alpha_{0}}{M-2 P+2 \operatorname{coth}(x-\lambda t)}
$$

with $p_{1}=2$.

For the third set (27), we obtain case I solutions with $P=1$ and $M=0$,

$$
\begin{aligned}
& \varphi_{15}(x, t)=\sqrt{k} \tan (\sqrt{k}(x-\lambda t)), \\
& \varphi_{16}(x, t)=\sqrt{k} \cot (\sqrt{k}(x-\lambda t)) .
\end{aligned}
$$

For $k<0$,

$$
\begin{gathered}
\varphi_{17}(x, t)=\sqrt{-k} \tanh (\sqrt{-k}(x-\lambda t)), \\
\varphi_{18}(x, t)=\sqrt{k} \operatorname{coth}(\sqrt{k}(x-\lambda t)),
\end{gathered}
$$

while for cases II-IV,

$$
\begin{gathered}
\varphi_{19}(x, t)=\frac{M}{2}-\sqrt{-k} P \operatorname{Tanh}[\sqrt{-k}(x-\lambda t)], \\
\varphi_{20}(x, t)=\frac{M}{2}-\sqrt{-k} P \operatorname{Coth}[\sqrt{-k}(x-\lambda t)], \\
\varphi_{21}(x, t)=\frac{M}{2}+\frac{1}{2}\left(-M-\frac{\sqrt{-1+r^{2}}+\operatorname{Sinh}[(x-\lambda t)]}{r+\operatorname{Cosh}[(x-\lambda t)]}\right) \\
\varphi_{22}(x, t)=\frac{M}{2}+\frac{1}{2}(M+\operatorname{Coth}[(x-\lambda t)]+\operatorname{Csch}[(x-\lambda t)]), \\
\varphi_{23}(x, t)=\frac{M}{2}+\frac{1}{2}(-M-2 \operatorname{Coth}[(x-\lambda t)]) .
\end{gathered}
$$

3.2. Example 2. Korteweg-de Vries Burgerss

Consider KdV-Burgerss equation [24,25],

$$
\varphi_{t}+\varphi \varphi_{x}+\alpha \varphi_{x x x}-\beta \varphi_{x x}=0 .
$$

Using the transformation $\varphi(x, t)=U(\eta), \eta=x-\lambda t$ we get the following ODE

$$
-\lambda U^{\prime}+U U^{\prime}+\alpha U^{\prime \prime \prime}-\beta U^{\prime \prime}=0 .
$$

Integrating (55) with respect to $\eta$ once, we get

$$
-\lambda U+\frac{1}{2} U^{2}-\beta U^{\prime}+\alpha U^{\prime \prime}=0,
$$

and balancing $U^{2}$ with $U^{\prime \prime}$ gives $\mathrm{n}=2$, thus, the solution should be in the form

$$
U=a_{0}+b_{0}+a_{1} Q+b_{1}(1+Q)^{-1}+a_{2} Q^{2}+b_{2}(1+Q)^{-2} .
$$

Substituting Equations (57) and (4) in Equation (56), we get a polynomial equation $Q$. Hence, equating the coefficient of $Q^{j}$ (i.e., $j=0,1,2, \ldots$ ) to zero and solving the obtained system of overdetermined algebraic equation using symbolic manipulation package Mathematica, we get the results: 
The first set:

$$
\begin{aligned}
k & =\frac{25 M^{2} \alpha^{2}-\beta^{2}}{100 P \alpha^{2}}, a_{1}=0, a_{2}=0, \\
b_{1} & =-\frac{12}{5}\left(-5 \alpha M^{2}+5 k \alpha M+15 P \alpha M-\beta M-10 P^{2} \alpha-10 k P \alpha+k \beta+P \beta\right), \\
b_{2} & =-12\left(\alpha k^{2}-2 M \alpha k+2 P \alpha k+M^{2} \alpha+P^{2} \alpha-2 M P \alpha\right), \\
\lambda & =\frac{25 M^{2} \alpha^{2}+300 P^{2} \alpha^{2}+200 k P \alpha^{2}-300 M P \alpha^{2}+30 M \beta \alpha-60 P \beta \alpha+25 a_{0} \alpha-\beta^{2}}{25 \alpha} .
\end{aligned}
$$

The second set:

$$
\begin{gathered}
P \alpha \beta \neq 0, k=\frac{25 M^{2} \alpha^{2}-\beta^{2}}{100 P \alpha^{2}}, a_{1}=-\frac{12}{5}(5 M P \alpha-P \beta), b_{1}=0, a_{2}=-12 P^{2} \alpha, b_{2}=0, \\
\lambda=\frac{25 M^{2} \alpha^{2}+200 k P \alpha^{2}-30 M \beta \alpha+25 a_{0} \alpha-\beta^{2}}{25 \alpha} .
\end{gathered}
$$

For the first set (58), if $M=0, P=1$ we get the solutions satisfying case I, for $k>0$, the solutions of KdV-Burgerss equation are

$$
\begin{aligned}
& \varphi_{1}(x, t)=a_{0}-\frac{12\left(\alpha k^{2}+2 \alpha k+\alpha\right)}{(\sqrt{k} \tan (\sqrt{k} \eta)+1)^{2}}-\frac{12(-10 k \alpha-10 \alpha+k \beta+\beta)}{5(\sqrt{k} \tan (\sqrt{k} \eta)+1)}, \\
& \varphi_{2}(x, t)=a_{0}-\frac{12\left(\alpha k^{2}+2 \alpha k+\alpha\right)}{(\sqrt{k} \cot (\sqrt{k} \eta)+1)^{2}}-\frac{12(-10 k \alpha-10 \alpha+k \beta+\beta)}{5(\sqrt{k} \cot (\sqrt{k} \eta)+1)} .
\end{aligned}
$$

For $k<0$,

$$
\begin{gathered}
\varphi_{3}(x, t)=a_{0}-\frac{12 \alpha(k+1)^{2}}{(\sqrt{-k} \tanh (\sqrt{-k} \eta)-1)^{2}}+\frac{12(10 \alpha-\beta)(k+1)}{5-5 \sqrt{-k} \tanh (\sqrt{-k} \eta)}, \\
\varphi_{4}(x, t)=a_{0}-\frac{12 \alpha(k+1)^{2}}{(\sqrt{k} \operatorname{coth}(\sqrt{k} \eta)-1)^{2}}+\frac{12(10 \alpha-\beta)(k+1)}{5-5 \sqrt{k} \operatorname{coth}(\sqrt{k} \eta)} .
\end{gathered}
$$

For $k=0$,

$$
\varphi_{5}(\eta)=\frac{12 \eta(\alpha+5 \beta(-2+\eta)-\alpha \eta)+5 A(-1+\eta)^{2} a_{0}}{5 A(-1+\eta)^{2}} .
$$

For cases II-IV, we apply the compatibility condition with the values of $M, k$ and $P$ in (26), we get $p_{1}=\frac{\beta}{5 \alpha}$, therefore, the solutions satisfying case II are

$$
\varphi_{6}(x, t)=a_{0}-\frac{12 \alpha(k+1)^{2}}{\left(p_{1} \tanh \left(\eta p_{1}\right)-1\right)^{2}}+\frac{12(10 \alpha-\beta)(k+1)}{5-5 p_{1} \tanh \left(\eta p_{1}\right)},
$$

and

$$
\varphi_{7}(x, t)=a_{0}-\frac{12 \alpha(k+1)^{2}}{\left(\operatorname{coth}\left(\eta p_{1}\right) p_{1}-1\right)^{2}}+\frac{12(10 \alpha-\beta)(k+1)}{5-5 \operatorname{coth}\left(\eta p_{1}\right) p_{1}}
$$

and for case III,

$$
\varphi_{8}(x, t)=a_{0}-\frac{48(k+1)^{2} \alpha(r+\cosh (\eta))^{2}}{\left(-2 r-2 \cosh (\eta)+\sinh (\eta)+\sqrt{r^{2}-1}\right)^{2}}-\frac{24(k+1)(10 \alpha-\beta)(r+\cosh (\eta))}{5\left(-2 r-2 \cosh (\eta)+\sinh (\eta)+\sqrt{r^{2}-1}\right)},
$$


with $p_{1}=1$, while for case IV, solutions will be in the form

$$
\varphi_{9}(x, t)=a_{0}-\frac{48 \alpha(k+1)^{2}}{(\operatorname{coth}(\eta)+\operatorname{csch}(\eta)+2)^{2}}+\frac{24(10 \alpha-\beta)(k+1)}{5(\operatorname{coth}(\eta)+\operatorname{csch}(\eta)+2)},
$$

with $p_{1}=1$,

$$
\varphi_{10}(x, t)=a_{0}-\frac{12\left(\alpha k^{2}+2 \alpha k+\alpha\right)}{(1-\operatorname{coth}(\eta))^{2}}-\frac{12(-10 k \alpha-10 \alpha+k \beta+\beta)}{5(1-\operatorname{coth}(\eta))}
$$

with $p_{1}=2$.

Similarly the first case of the second set (59), the solutions are:

for $k>0$,

$$
\begin{aligned}
& \varphi_{11}(x, t)=a_{0}-12 k \alpha \tan ^{2}(\sqrt{k} \eta)+\frac{12}{5} \sqrt{k} \beta \tan (\sqrt{k} \eta), \\
& \varphi_{12}(x, t)=a_{0}-12 k \alpha \cot ^{2}(\sqrt{k} \eta)+\frac{12}{5} \sqrt{k} \beta \cot (\sqrt{k} \eta),
\end{aligned}
$$

while for $k<0$,

$$
\begin{gathered}
\varphi_{13}(x, t)=a_{0}+12 k \alpha \tanh ^{2}(\sqrt{-k} \eta)-\frac{12}{5} \sqrt{-k} \beta \tanh (\sqrt{-k} \eta), \\
\varphi_{14}(x, t)=a_{0}-\frac{12}{5}\left(5 k \alpha \operatorname{coth}^{2}(\sqrt{k} \eta)+\sqrt{k} \beta \operatorname{coth}(\sqrt{k} \eta)\right),
\end{gathered}
$$

and for $k=0$,

$$
\varphi_{15}(\eta)=a_{0}-\frac{12(5 \beta+\alpha \eta)}{5 A \eta^{2}} .
$$

As we did previously, we apply the compatibility condition (28) from (59), and we get $p_{1}=\frac{\beta}{5 \alpha}$. Hence, case II solutions are:

$$
\varphi_{16}(x, t)=a_{0}-3 \alpha p_{1}^{2}\left(M+2 \tanh \left(\eta p_{1}\right)\right)^{2}+\frac{6}{5}(5 M \alpha-\beta) p_{1}\left(M+2 \tanh \left(\eta p_{1}\right)\right),
$$

and

$$
\varphi_{17}(x, t)=a_{0}-3 \alpha\left(M+2 \operatorname{coth}\left(\eta p_{1}\right)\right)^{2} p_{1}^{2}+\frac{6}{5}(5 M \alpha-\beta)\left(M+2 \operatorname{coth}\left(\eta p_{1}\right)\right) p_{1},
$$

and for case III,

$$
\varphi_{18}(x, t)=a_{0}-3 \alpha\left(M+\frac{\sinh (\eta)+\sqrt{r^{2}-1}}{r+\cosh (\eta)}\right)^{2}+\frac{6(5 M \alpha-\beta)\left(M r+M \cosh (\eta)+\sinh (\eta)+\sqrt{r^{2}-1}\right)}{5(r+\cosh (\eta))},
$$

where $p_{1}=1$, while case IV solutions are

$$
\varphi_{19}(x, t)=a_{0}-3 \alpha(M+\operatorname{coth}(\eta)+\operatorname{csch}(\eta))^{2}-\frac{6(5 M P \alpha-P \beta)(M+\operatorname{coth}(\eta)+\operatorname{csch}(\eta))}{5 P},
$$

with $p_{1}=1$.

$$
\varphi_{20}(x, t)=a_{0}+\frac{3}{5}(M+2 \operatorname{coth}(\eta))(5 M \alpha-10 \operatorname{coth}(\eta) \alpha-2 \beta),
$$

with $p_{1}=2$.

\section{Discussion}

The extended HB method has been applied for deriving many analytical wave solutions for foam drainage equation and KdV-Burgers equation. The obtained solutions cover different types, like shock, periodical, rational, solitary, singular and explosive wave solutions. For example, solutions (45) and 
(70) are sinusoidal-type periodical solutions shown in Figure 1, and the rational solutions are (39) and (74), which may be helpful to explain certain physical phenomena, while solutions (50) and (73) are explosive/blow up solutions [1,34] (see Figure 2). The solutions (49) and (72) are shock wave solutions as depicted in Figure 3, note here that the shock wave solution of the foam drainage equation has an important role to describing the motion in the foam while the shock wave solution of the KdV-Burgers equation is very important for studying the acoustic waves in plasma physics as in $[29,30]$ where the dust acoustic shock waves have been studied. The solution (77) represents a soliton wave (Figure 4) which plays a great role in the study of plasma physics [35-37].

Zayed and Al-Nwehy [31] solved the foam drainage equation using $G^{\prime} / G$ method and KdV-Burgers equation were solved in [16,25-27]. On comparing their results with our obtained results, we can see that we obtained the same solutions in addition to many new solutions.

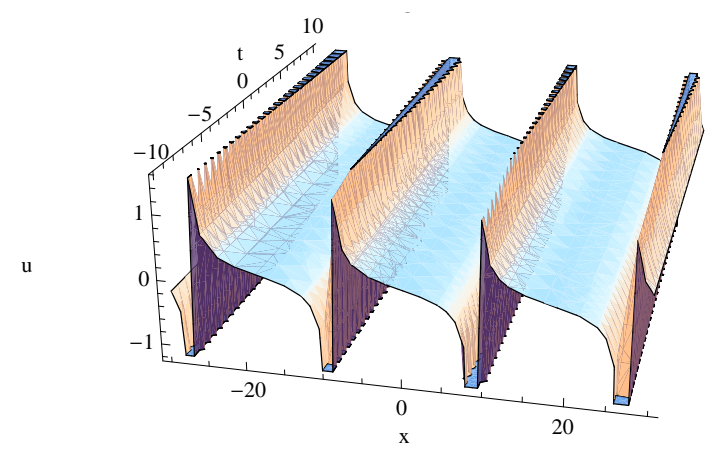

Figure 1. 3D Plot of the periodical solution (45), with $k=0.1$ and $\lambda=-0.1$.

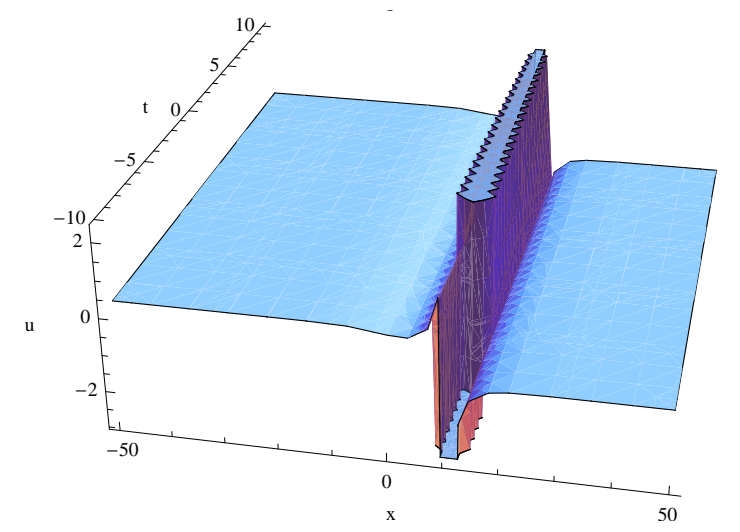

Figure 2. 3D Plot of the explosive solution (30), with $M=0.4$ and $\lambda=0.1$.

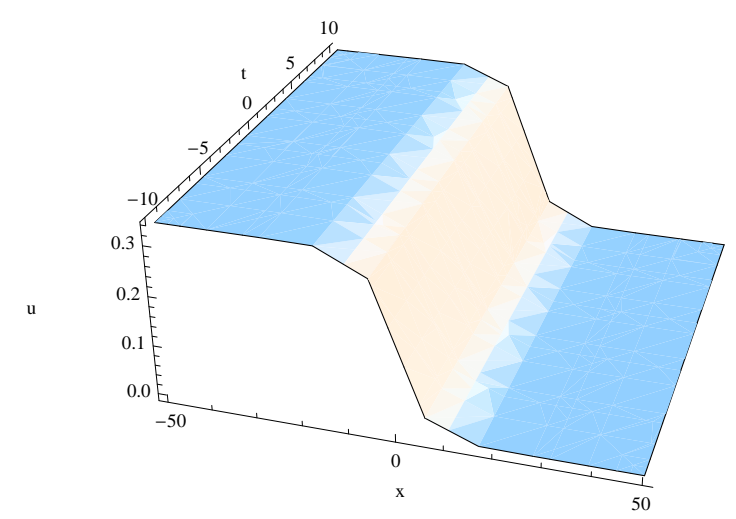

Figure 3. 3D Plot of the shock wave solution (49), with $k=-0.1, M=0.4$ and $\lambda=0.1$. 


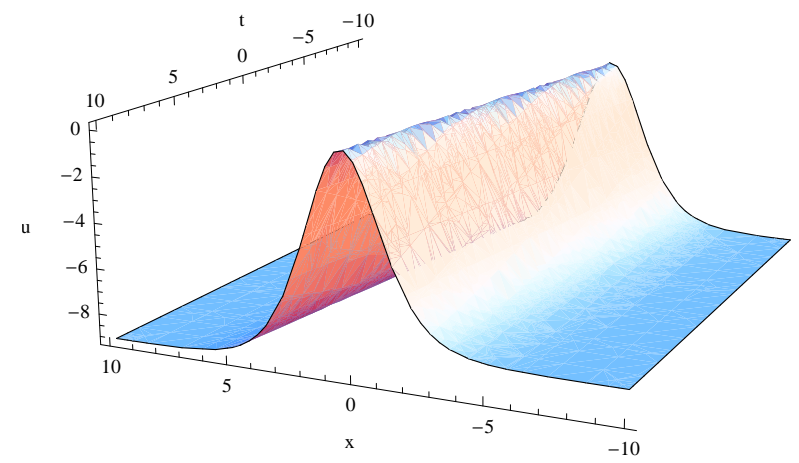

Figure 4. 3D Plot of the soliton solution (77), with $r=1.2$ and $M=0.1$.

\section{Conclusions}

In summary, the extended HB method has been developed and applied with the help of Mathematics to deal with nonlinear partial differential equations (PDEs). The traveling wave solutions were formally derived for the foam Drainage Equation and the KdV-Burgers equation. The approach is an advanced tool and more general than many methods like tanh or extended tanh methods to give new and rich variety of solutions for nonlinear evolution equations. These different types of solutions play an important role in studying many physical phenomena.

Author Contributions: All authors contributed equally and significantly in writing this article.

Funding: This research received no external funding.

Conflicts of Interest: The authors declare no conflicts of interest.

\section{References}

1. Moslem, W.M.; Sabry, R.; Abdelsalam, U.M.; Kourakis, I.; Shukla, P.K. Solitary and blow-up electrostatic excitations in rotating magnetized electron-positron-ion plasmas. N. J. Phys. 2009, 11, 033028. [CrossRef]

2. Abdelsalam, U.M.; Allehiany, F.M.; Moslem, W.M.; El-Labany, S.K. Nonlinear structures for extended Korteweg-de Vries equation in multicomponent plasma. Pramana J. Phys. 2016, 86, 581-597. [CrossRef]

3. Abdelsalam, U.M.; Zobaer, M.S. Exact Traveling Wave Solutions of KdV Equation for DAWs in Superthermal Plasma. Rev. Mex. Astron. Astrofsica 2018, 54, 363-373.

4. Abdelsalam, U.M. Traveling wave solutions for shallow water equations. J. Ocean. Eng. Sci. 2017, 2, $28-33$. [CrossRef]

5. Baskonus, H.M.; Sulaiman, T.A.; Bulut, H. On the new wave behavior to the Klein-Gordon-Zakharov equations in plasma physics. Indian J. Phys. 2019, 93, 393-399. [CrossRef]

6. Drazin, P.G.; Johnson, R.S. Solitons: An Introduction; Cambridge University Press: Cambridge, UK, 1989.

7. Vakhnenko, V.O.; Parkes, E.J.; Morrison, A.J. A Backlund transformation and the inverse scattering transform method for the generalised Vakhnenko equation. Chaos Solitons Fractals 2003, 17, 683-692. [CrossRef]

8. Hirota, R. Direct method of finding exact solutions of nonlinear evolution equations. In Backlund Transformations; Bullough, R., Caudrey, P., Eds.; Springer: Berlin, Germany, 1980; pp. 1157-1175.

9. Lu, D.; Hong, B.; Tian, L. Backlund Transformation and N-soliton-like Solutions to the Combined KdV-Burgers Equation with Variable Coefficients. Int. J. Nonlinear Sci. 2006, 10, 3-10.

10. Malfliet, W. Solitary wave solutions of nonlinear wave equations. Am. J. Phys. 1992, 60, 650-654. [CrossRef]

11. Gao, Y.T.; Tian, B. Generalized tanh method with symbolic computation and generalized shallow-water wave-equation. Comput. Math. Appl. 1997, 33, 115-118. [CrossRef]

12. Fan, E. Extended tanh-function method and its applications to nonlinear equations. Phys. Lett. A 2000, 277, 212-218. [CrossRef]

13. Abdelsalam, U.M. Exact travelling solutions of two coupled $(2+1)$-Dimensional Equations. J. Egypt. Math. Soc. 2017, 25, 125-128. [CrossRef] 
14. El-wakil, S.A.; Madkour, M.A.; Abdou, M.A. Application of Exp-function method for nonlinear evolution equations with variable coefficients. Phys. Lett. A 2007, 369, 62-69. [CrossRef]

15. Wang, M.L.; Li, X.; Zhang, J. The (GG)-expansion method and travelling wave solutions of nonlinear evolution equations in mathematical physics. Phys. Lett. A. 2008, 372, 417. [CrossRef]

16. Abdelsalam, U.M.; Zobaer, M.S. Exact Traveling Wave Solutions of Further Modified Korteweg-De Vries Equation in Multicomponent Plasma. Iran. J. Sci. Technol. Trans. Sci. 2018, 42, 2175-2182. [CrossRef]

17. Abdelsalam, U.M.; Selim, M. Ion acoustic waves in a degenerate multicomponent magnetoplasma. J. Plasma Phys. 2013, 79, 163. [CrossRef]

18. Sabry, R.; Zahran, M.A.; Fan, E. A new generalized expansion method and its application in finding explicit exact solutions for a generalized variable coefficients KdV equation. Phys. Lett. A 2004, 326, 326-393. [CrossRef]

19. Moslem, W.M.; Abdelsalam, U.M.; Sabry, R.; Shukla, P.K. Electrostatic structures associated with dusty electronegative magnetoplasmas. N. J. Phys. 2010, 12, 73010. [CrossRef]

20. Moussa, M.H.M.; El-Shiekh, R.M. Similarity reduction and similarity solutions of Zabolotskay- Khoklov equation with dissipative term via symmetry method. Phys. A 2006, 371, 325-335. [CrossRef]

21. Wang, M.L. Solitrary wave solution for variant Boussinesq equation. Phys. Lett. A 1995, 199, $169-172$. [CrossRef]

22. Wang, M.L. Applicatian of homogeneous balance method to exact solutions of nonlinear equation in mathematical physics. Phys. Lett. A 1996, 216, 67-75. [CrossRef]

23. Fan, E.G.; Zhang, H.Q. New exact solutions to a system of coupled KdV equations. Phys. Lett. A 1998, 245, 389-392. [CrossRef]

24. Yang, L.; Zhu, Z.; Wang, Y. Exact Solutions of Nonlinear Equations. Phys. Lett. 1999, 260, 55-59. [CrossRef]

25. Fan, E.G. Two new applications of the homogeneous balance method. Phys. Lett. A 2000, 265, 353-357. [CrossRef]

26. Verbist, G.; Weaire, D.; Kraynik, A.M. The foam drainage equation. J. Phys. Condens. Matter 1996, 8, 3715. [CrossRef]

27. Meng, P.; Yin, W. The Travelling Wave Solutions of KdV-Burgers Equations. In Proceedings of the International Conference on Management Science and Innovative Education (MSIE2015), Xi'an, China, 12-13 December 2015.

28. Senthilkumaran, M.; Selvam, S.K. Solitary wave solution of the variable coefficient KdV-Burgers equation. Bull. Soc. Math. Banja Luka 2017, 7, 395-401.

29. Chukkol, Y.B.; Mohamad, M.N.; Muminov, M.I. Exact Solutions to the KDV-Burgers Equation with Forcing Term Using Tanh-Coth Method. Aip Conf. Proc. 2017, 1870, 040024.

30. Zaghbeer, S.K.; Salah, H.H.; Sheta, N.H.; El-Shewy, E.K.; Elgarayhi, A. Dust acoustic shock waves in dusty plasma of opposite polarity with non-extensive electron and ion distributions. J. Plasma Phys. 2014, 80, 517-528. [CrossRef]

31. Nakamura, Y.; Bailung, H.; Shukla, P.K. Coulomb Dissociation of ${ }^{19} \mathrm{C}$ and its Halo Structure. Phys. Rev. Lett. 1999, 83, 1602-1605. [CrossRef]

32. Masood, W.; Rizvi, H.; Jehan, N.; Siddiq, M. Dissipative cylindrical fast magnetoacoustic waves in planetary magnetospheres. Astrophys. Space Sci. 2011, 335, 405-413. [CrossRef]

33. Zayed, E.M.E.; Al-Nowehy, A.G. Exact solutions for nonlinear foam drainage equation. Indian J. Phys. 2017, 91, 209-218. [CrossRef]

34. Abdelsalam, U.M.; Allehiany, F.M.; Moslem, W.M. Nonlinear Waves in GaAs Semiconductor. Acta Phys. Pol. A 2016, 129, 472-477. [CrossRef]

35. Abdelsalam, U.M. Dust-ion-acoustic solitary waves in a dense pair-ion plasma. Phys. B 2010, 405, $3914-3918$. [CrossRef] 
36. Abdelsalam, U.M. Solitary and freak waves in superthermal plasma with ion jet. J. Plasma Phys. 2013, 79, 287-294. [CrossRef]

37. Abdelsalam, U.M.; Allehiany, F.M. Different Nonlinear Solutions of KP Equation in Dusty Plasmas. Arab. J. Sci. Eng. 2018, 43, 399-406. [CrossRef]

(C) 2019 by the authors. Licensee MDPI, Basel, Switzerland. This article is an open access article distributed under the terms and conditions of the Creative Commons Attribution (CC BY) license (http:/ / creativecommons.org/licenses/by/4.0/). 